\title{
The Effect of Induced Multipoles on the Fifth-order Raman Response
}

\author{
Thomas I. C. Jansen, Koos Duppen, and Jaap G. Snijders \\ Theoretical Chemistry, ${ }^{\dagger}$ Cltrafast Laser Laboratorv, Materials Science Centre. Rijhsuniversiteit Groningen (RuG). \\ Nijenborgh 4. 9747.AG Groningen, The Netherlands \\ Received.April 17, 2003
}

\begin{abstract}
In our previous work we developed the Finite Field method in order to calculate the fifth-order Raman response. The method was applied to calculate various polarization components of the two-dimensional response of liquid $C S_{乏}$. So far, all calculations relied on the dipole-induced dipole. Accurate time-dependent density functional theory calculations have shown that this model has big discrepancies. when molecules are close together as in the liquid. We now report results of investigations on the importance of multipole and electron overlap effects on the polarizability and the fifth-order Raman response. It is shown that these collision effects. especially the induced multipoles are crucial in the description of the fifth-order response. The impact is found to be especially pronounced for the $\chi_{m m: z}^{(5)}$. response that is solely due to interaction induced effects. The calculated response will be compared with various experimental results.
\end{abstract}

Key Words : Induced multipoles, Fifth-order Raman, Finite field nethod

\section{Introduction}

Many chemical reactions take place in the liquid phase and these reactions are highly influenced by the dynamics of the solvent. Therefore the dynamics of liquids have been studied extensively experimentally applying, for example. third-order time resolved Raman teclniques. ${ }^{1.6}$ In the last decade substantial efforts have been made in order to measure, ${ }^{7.15}$ model ${ }^{16.23}$ and calculate ${ }^{2.4 .41}$ the fifth-order Raman response suggested by Tanimura and Mukamel. ${ }^{16}$ Spectroscopies relying on this response should be able to distinguish between different relaxation mechanisnis. which the lower order techniques calunot. Furthernore, the fifth-order response is also sensitive to mode coupling ${ }^{17-19}$ and anhamonicities.

The fifth-order response has shown to be a great clallenge to measure. In these experinents the sample is first pumped by a pair of laser pulses. After a time delay $t_{1}$ two mixing pulses are applied and finally after a second time delay $t_{\Sigma}$ a probe pulse is applied and the fifth-order signal measured. So far only measurements have been performed on liquid carbon disulfide that has a large anisotropy in the molecular polarizability. This leads to a very strong anisotropic thirdorder Raman response arising from the orientational motion. Unfortunately this strong third-order response leads to serve contamination with so called cascaded response in the fifthorder measurements. ${ }^{10.39}$ These cascaded processes consists of two third-order processes taking place at two different places in the liquid where the signal from one of these processes acts as a pump or probe pulse in the other process. Since these cascaded processes arise from the exact same electric field sequence as the true fifth-order response they have the same overall phasematching conditions. ${ }^{11}$ making them extremely difficult to suppress. Heterodyne detection tecluniques ${ }^{11.15}$ and multi-color experiments ${ }^{10.14}$ have been applied in order to overcome these problems.

The third- and fifth-order Raman responses are. however. not only due to the single molecule reorientational motion. Since the molecules in a liquid are closely packed and constantly touch each other interaction induced polarizabilities play an important role. These polarizabilities arise when local fields are generated so the molecules do not only feel the macroscopic field in the sample arising from the applied laser fields, but also fields generated from dipoles induced on all the other molecules in the surrounding. ${ }^{+2,+3}$ This means that intermolecular motion also changes the overall polarizability and that a response will also arise from the intermolecular motion. The polarizability is thus really a molecular property and in a true liquid we ought to consider its macroscopic counterpart, the susceptibility. ${ }^{\text {th }}$

Real molecules are not just point like polarizabilities giving rise to point dipoles. The molecular polarizability is an electronic property and the electrons are spread out all over the molecule. This give rise to multipole effects on the susceptibility since for example in carbon disulfide most of the electrons are located on the sulfur atoms. Since the electrons are not located at the atomic centers. but rather spread out in a cloud around these the susceptibility is also affected by overlapping electron clouds of neighbouring molecules. ${ }^{42.45,46}$ In previous work we investigated these effects in the interaction between carbon disulfide molecules and their impact on the third-order response. ${ }^{47}$ Both effects from the dipole-induced dipole effect and the induced multipole effects. where found to affect the third-order response. In dimers the polarizability at short ranges as those found in the first solvation shell in carbon disulfide the electron cloud overlap effects were also found to be significant. while their effect on the third-order response were less pronounced. In sinilar investigations on liquid xenon the effects of the electron cloud overlap on the third-order response was, however, found to be quite significant. ${ }^{48}$

Various methods exists to calculate the fifth-order Raman response from molecular dynamics simulations. These methods 
can be divided into two types. those approximating the motion in some way before calculating the response and those taking the full motion into account. Normal mode approaches ${ }^{-1.28 .3437}$ mode coupling methods ${ }^{-5.33}$ and the GLE approach $^{38}$ all belong to the first type and their success depends on whether the dynamic information they exclude is superfluous or not. The time correlation function method ${ }^{25.88}$ and the finite field method ${ }^{3 .+40}$ on the other hand both take all motion into account. The first of these methods is an equilibrium method that allows the calculation of any imaginable response depending on the molecular motion. whereas the second method is a non-equilibrium simulation of one specific experiment. The later method has the advantage that it is much faster than the first. because it is only taking the dynamic information into account that is needed in order to treat the specified experiment of interest. This corresponds to propagating one single vector of the so called stability matrix $=4.39 .40 .49$ while the full stability matrix is propagated in the time correlation function method.

In the following section the models used in order to calculate the susceptibility are described. In section the molecular dynamics simulations are described. The results are presented in section together with a discussion. Finally in section the conclusions are presented.

\section{Modeling the Susceptibility}

The first-order susceptibility will be approximated by a hierarchy of models. where each model includes the interaction induced effects on a different level of complexity: The molecular model (MOL) only accounts for the contributions from single molecule polarizabilities..$^{39}$ Local fields generated by induced dipoles on neighboring molecules are taken into account in the dipole-induced dipole model (DID). In most

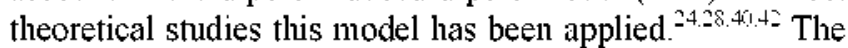
extended structure of the molecules is accounted for by using a model with atomic polarizabilities (POL) instead of molecular ones. Similar models have been used in other studies of the third- and fifth-order Raman response 35.010 .51

The effect of overlapping electron clouds is taken into account in an approximate way by using the direct reaction field model (DRF) ${ }^{43.45}$ In this model a set of screening functions damp the interaction as the atoms start overlapping. assuming that the electron clouds are well described by exponentially decaying densities.

The decay rate for the electron cloud and the atomic polarizabilities in the DRF model were chosen to exactly reproduce the two components of the dipole polarizability of a single molecule. They were optimized in order to give as good agreement with time-dependent density functional theory' (TDDFT) calculations on various dimers. ${ }^{47}$ The two components of dipole-octupole polarizability. which is the first non-vanishing multipole polarizability was also calculated using TDDFT and can be compared to the one obtained for a single molecule in the DRF model. The isotropic polarizability used for carbon disulfide is $8.95 \mathrm{~A}^{3}$. and the anisotropic polarizability is $10.05 \mathrm{~A}^{3}$. The dipole-octupole polarizabilties found with the TDDFT for the two components $\alpha_{=}^{3(1)}$ and $\alpha_{=}^{31 c}$ using the notation of Stone ${ }^{52}$ were 53.03 $\mathrm{A}^{5}$ and $29.29^{\circ} \mathrm{A}^{5}$ respectively. These dipole-octupole polarizabilities are found to be $81.53 \mathrm{~A}^{5}$ and $30.93 \mathrm{~A}^{5}$ in the DRF model applied. All these models and calculations have been described more thoroughly in our earlier paper considering the effects in the third-order Raman response. ${ }^{47}$

In order to interpret the two-dimensional spectra the response have been calculated using these four different models and comparing the results allows to examine the origin of the fifth-order response.

\section{Simulations}

The finite field method ${ }^{39-41}$ was used in order to calculate the fifth-order response. A simulation box containing 64 carbon disulfide molecules was used. Compared to earlier calculations with 256 molecules employing the DID method no significant change was observed when we limited ourselves to using 64 molecules. Because the simulation box is much smaller than the wavelength of the laser light the simulations are on a microscopic level not taking the wave-vectors $(\vec{k})$ into account. The simulations can therefore be seen to be performed at perfect phase matching conditions and the different kinds of response are separated depending on. how they scale with the strength of the laser fields. Cascaded processes are. however. never introduced in the calculations since the intermediate fields are not included

In the calculations a simple Lenuart-Jones force field optimized to give the correct thermodynamical properties was used ${ }^{53}$ The isothermal-isobaric ensemble was employed at $298 \mathrm{~K}$ and $\mathrm{l}$ bar using the procedures by Berendsen $e t$ al. ${ }^{54}$ Calculations with a constant energy' instead of temperature did not show any difference and when varying the time constant in the pressure conserving scheme no changes in the response was observed in test calculations along the $t_{2}$ axis. The used timesteps in the integration was $10 \mathrm{fs}$ and the carbon disulfide molecules were kept rigid. In order to collect sufficient statistical data the response was calculated using 4000 different starting configurations and the response was calculated for $t_{1}$ and $t z$ between 0 and $600 \mathrm{fs}$ with a $20 \mathrm{fs}$ resolution. In the calculations with the DRF model a laser field strength of $1.915 \mathrm{~V} / \mathrm{A}$. while a slightly lower value of $1.724 \mathrm{~V} / \mathrm{A}$ was used for the MOL. DID and POL models. In the case of DID only 2000 different starting configurations were needed. All responses were investigated in a broad range of laser field strengths in order to ensure both a good signal to (numerical) noise ratio and avoid contamination with higher-order Raman processes.

These conditions were used in order to calculated the $x_{i}^{(5)}=z$ and $x_{n m z}^{(5)} ; z$ responses. where $z$ denotes a polarization of the individual laser field along the $z$-axis while $m$ denotes a polarization along an axis forming the magic angle of $54.7^{\circ}$ with the $z$-axis. In the $\chi_{m m z=z}^{(5)}$ response this is equivalent with first pumping with $z$ polarized lasers. waiting the delay time $t_{1}$ and then pumping again with $z$ polarized lasers and 
then finally after the second delay time measuring the effect on the isotropic susceptibility.

\section{Results and Discussion}

In Figure 1 and 2 the $\chi^{(5)}$ shown respectively. For the $\chi=$ component the molecular response (MOL) depending only on the reorientational motion stretches somewhat out along the $t_{-}$-axis. The response including dipole-induced dipole interaction between the molecules and hence containing contributions from the intermolecular motion is much more symmetric along the two time axes. The response including the induced multipoles (POL) is again more stretched out along the $t$-axis. This is probably partially because the dipole-induced dipole model overestimates the interaction-induced effects. Including the electron overlap effect (DRF) changes the response only slightly.

The $\chi_{m m z s: s}^{(5)}$ response component is especially interesting since the change in the isotropic susceptibility is measured as explained in the end of last section. This means that the single molecule reorientation does not contribute to the spectrum and the (MOL) response vanishes. Therefore only the DID. POL and DRF responses are shown in Figure 2.
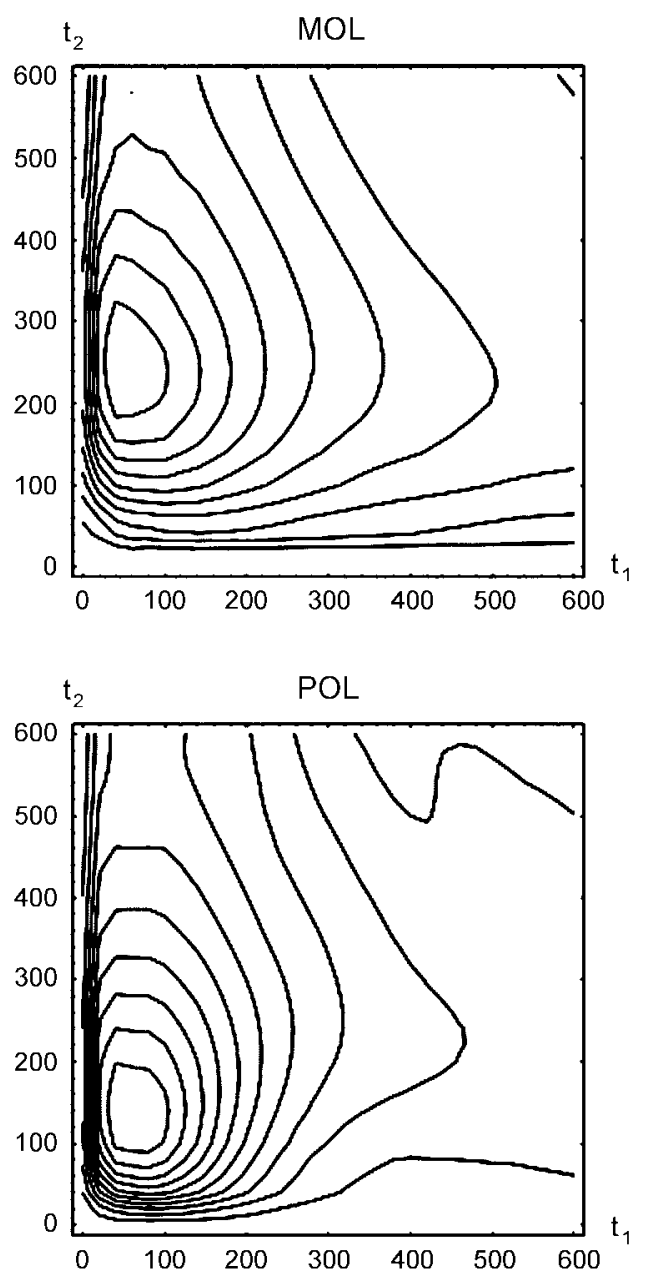

Figure 1. The all polarized two-dimensional Ramann response $\chi_{\text {is }}^{i \text { i }}$
This component therefore offers a unique opportunity to investigate the interaction-induced effects. The dipoleinduced dipole response is found to be rather symmetrical in the two time coordinates just as for the $\chi_{z=z:}^{(5)}$ component. When the induced multipoles (POL) are included the response changes significantly and becomes a ridge along the $t_{2}$ axis. however. a small peak is still observed. Including the electron cloud overlap the ridge is preserved. but the little peak is further diminished.

From the above observations we can conclude that several different kinds of motion contribute to the response. The liquid motion does not depend on which susceptibility model is used but which motion contributes and with what strength is determined by the susceptibility model. First a contribution from the pure molecular reorientation is found. Further two different kinds of response involving inter molecular motion are observed. One giving rise to the peak like structure observed when the DID is used and another giving rise to the ridge like structure that is observed. when the POL and DRF models are used. It will be interesting to do further investigation of the origin of these two last kinds of response.

Several experiments have been performed on the $\chi_{i=3}^{(5)}$ response. Different results have been reported. In the
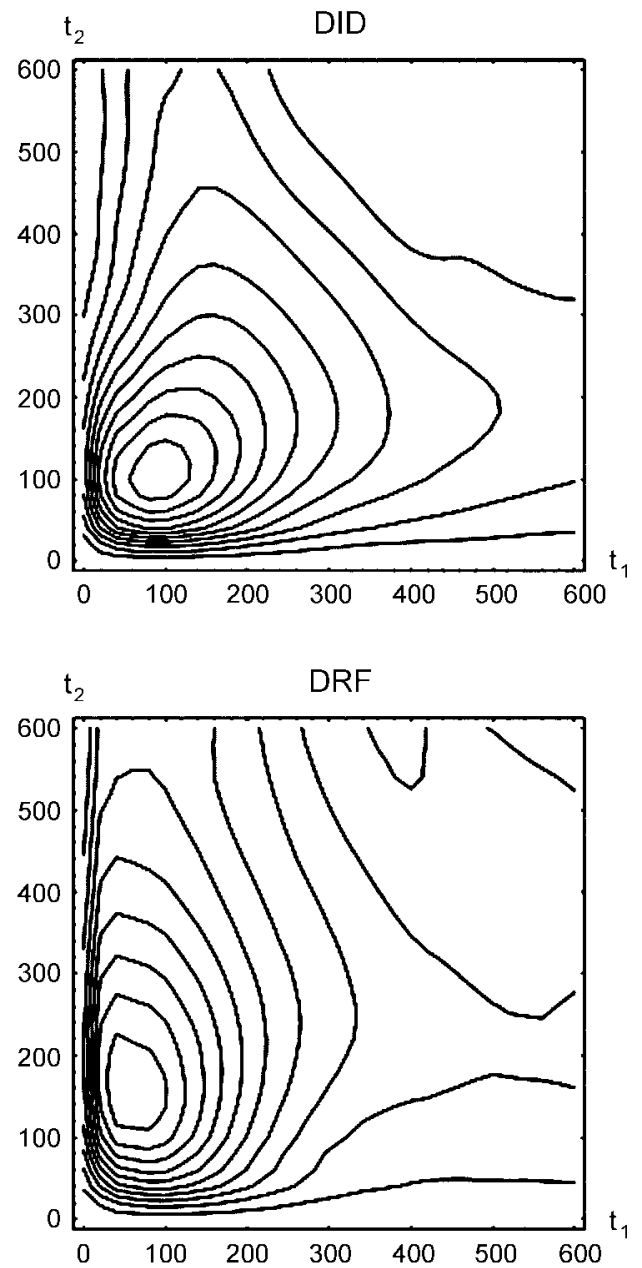

in the MOL, POL, DID and DRF models. The time is given in $\mathrm{fs}$. 

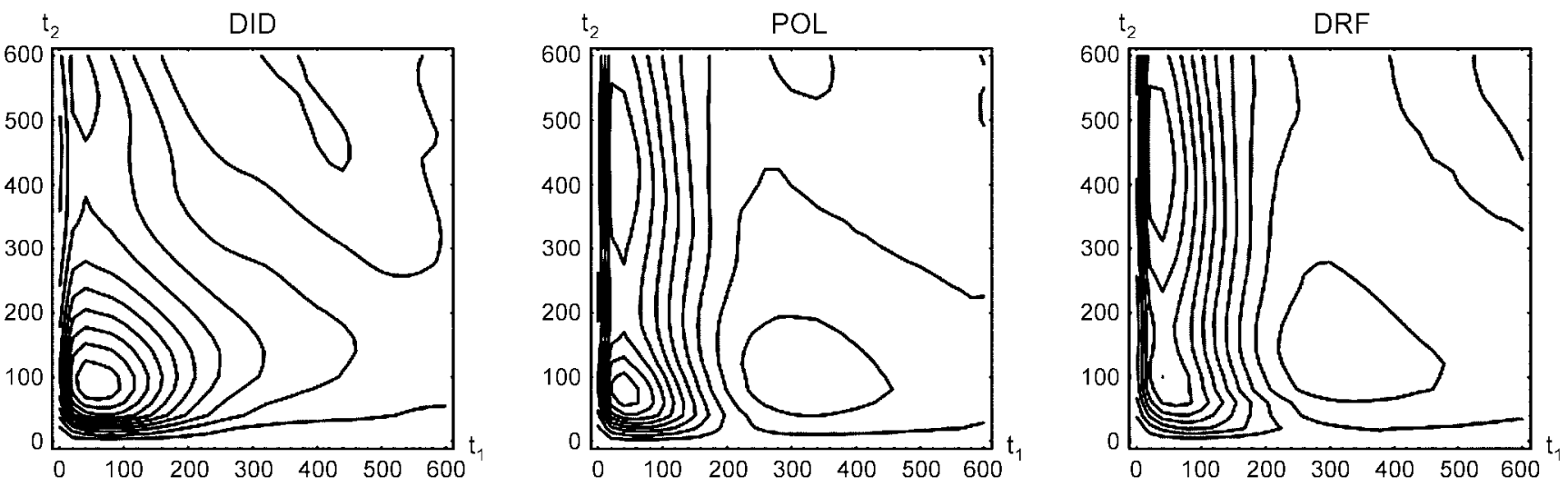

Figure 2. The $\chi_{m m z z z}^{i s 1}$ two-dinensional Raman response in the DID, POL and DRF models, where $m$ denotes the magic angle compared to the $z$-axis. The time is given in $\mathrm{fs}$.

complicated multi-color experiments reported by Kubarych et $a^{12-1+55.56}$ a clear ridge along the $t=$-axis was found. The time evolution of this ridge agree well with our observations. On the other hand Kaufman et $\mathrm{ct}^{15}{ }^{15}$ reported a node on the $t_{\mathbf{S}^{-}}$ axis. which is not consistent with our observations. On the other hand these experiments are backed up by calculations performed by Saito et $a l^{28}$ There can however. only be one correct experimental response and theoretical calculations under the same conditions should give the same result. This problem should therefore be resolved.

There are. however. several differences between our calculations and the calculations performed by Saito and Ohmine ${ }^{38}$ who used the NVE (microcanonical) ensemble at a temperature around $270 \mathrm{~K}{ }^{57}$ They used the time correlation function method and only 32 molecules. Further their calculations only included the DID model and their results should be compared with our DID results. The node they reported arose already in the single molecule reorientational response. but we observe no nodes in any susceptibility model. The low temperature or the ensemble they use could maybe result in the observed nodes. To explain this theoretical inconsistency the simulations should be performed at the exact same conditions and preferably at conditions as close as possible to those found in the experiments.

In order to solve the experimental controversy an agreement between the theoretical results would certainly help. On the other hand performing experiments on other liquids could also provide help. Since the molecular anisotropy in the polarizability of carbon disulfide is so large the response from this liquid is also very strong as noted in the introduction. This has been the most important reason to use this liquid. On the other hand this has also resulted in problems with intense cascading response and since the cascading response involve four Raman events and the true fifth-order response involve only three. Therefore. the cascading response will disappear faster than the true fifth-order response, when the polarizablity fluctuations in the liquid are lowered. Therefore. the problems with cascading processes can be expected to be smaller in other liquids than carbon disulfide. When the polarizability fluctuations are lower the intensity of the fifth-order signal will of course also be lower and this may present a problem with the signal to noise ratio in the experiments. Liquids like xenon. benzene and carbon tetrachloride could be candidates for new experiments.

\section{Conclusions}

It was demonstrated that both induced multipole effects and electron overlap effects are very important in the fifthorder response of liquid carbon disulfide. The different models applied reveal contributions from different kinds of motion which should be studied more carefully. The calculated $\chi_{y=2}^{(3)}$, response was found to be in good agreement with the experimental observations by Kubarych et al. ${ }^{12-1+55.56 \mathrm{i}}$ The smaller deviations might very well arise because the LennartJones force field we use is too simple. The fifth-order Raman response might therefore show to be a sensitive tool to investigate both interaction induced effects and intermolecular forces and motion in the liquid phase. This will of course require that consensus is reached about which of the different experiments reveal the true fifth-order Raman response.

Still further research should be performed in order to get a deeper understanding of the fifth-order Raman response of liquid carbon disulfide and allow a clear interpretation of the spectra.

\section{References}

1. Ruhman. S: Wiliams, L. R.: Joly, A. G.: Nelson, K. A. J. Plys. Chem 1987. 91.2237.

2. Waldman. A.: Baninn. U.: Rabani. E.: Ruhmant. S. J. Phns Chent. 1992.96. 10840 .

3. MeMorrow, D.: Thantu, N.; Melinger. J. S.; Kim. S. K.; Lotshaw, W. T. J. Plws. Chem 1996. 100, 10389.

4. Steffen. T; Meinders, N. A. C. M.; Duppen, K. J. Plys. Chem. A 1998. 102. 4213.

5. Steffert. T.: Duppent. K. J. Chem. Phys. 1997. 106.3854.

6. McMorrow. D.: Thantu. N.: Kleiman. V.: Melinger. J. S.: Lotshaw. W. T. J. Ph.s. Chent A 2001. 105(34). 7960.

7. Steffen. T.: Duppen. K. Plys. Rav Lett. 1996, 76, 1224.

8. Tokmakoff, A.; Fleming, G. R. J. Chem. Phus. 1997, 106, 2569.

9. Tominaga. K.: Yoshihara. K. J. Chem. Plys. 1996. 104. 4419.

10. Blank. D. A.: Kaufman. L. J.: Flening. G. R. J. Chent Phos. 1999. .111(7). 3105 .

11. Golonzka, O.: Demirdöven. N.: Khalil, M: Tokmakoff, A. $J$. 
Chem. Phys. 2000. H3(22), 9893 .

12. Astinov, V: Kubarych. K. I: Milne. C. I.: Miller. R. I. D. Optics Letters 2000. $25(1 \mathrm{i}) .853$.

13. Astinov. A.: Kubarych. K. J.: Milne. C. J.: Dwayne Miller. R. J. Chem. Phvs. Lett. 2000. 327.334

14. Kubarych. K. I.: Milne, C. J. Lin. S: Astinot. V: Miller. R. I. D. J. Chent. Phvs. 2002. I6. 2016

15. Kaufman L. J: Heo, J.: Ziegler, L. D.: Fleming G. R. Plys. Rev Lett. 2002. 88.207402.

16. Tanimura. Y: Mukamel. S. J. Chem. Phys. 1993. 99.9496.

17. Okumura. K.: Tanimura. Y.J. Chem. Phws. 1997.107.2267.

18. Okumura, K.: Tokmakoft. A.; Tanimura. Y. J. Chem. Phos. 1999. 111,492 .

19. Tokmakoff. A: Lang, M. J.: Larsen, D. S.: Fleming, G. R.: Chermyak. V: Mukamel. S. Phys. Rev: Lett. 1997. 79. 2702.

20. Okumura. K.: Tanimura. Y. J. Chem. Phys. 1997.106. 1687.

21. Steffen. T.: Duppen. K. Chent. Phns. 1998. 233. 267.

22. Steffen, T: Tanimulua. Y. J. Phus Soc. Jp. 2000. $69(9), 3115$.

23. Tanimura, Y: Steffen. T. J. Phis Soc. Jp. 2000. $69(12), 4095$

24. Saito, S Ohmine, I. J. Chem Phys. 1998. 108, 240.

25. Ma. A.: Stratt. R. M. J. Chem. Plns. 2002. 116.4972.

26. Ma. A.: Stratt. R. M. Pln's. Rev: Lett. 2000. 85. 1004.

27. Ma. A.: Stratt. R. M. J. Chem. Phns. 2000. 116.4962.

28. Saito, S.: Ohninie, I. Phns. Rev Lett 2002, 88. 207401.

29. Denny. R. A.: Reichman. D. R. Phvs. Rev E. 2001, 63,065101.

30. Demy. R. A.: Reichman. D. R. J. Chem. Plys. 2002. 116, 1987.

31. van Zon. R.: Schofield. J. Phys. Rev. E 2002. 65.011106.

32. van Zon. R.: Schofield. J. Plys. Rev. E 2002. 65.011107.

33. Rabani. E.: Reichman. D. R. PHws. Rev: E 2002, 65.036111.

34. Cao J.: Wh. J. Yang S. J. Chem Phus. 2002, 116, 3760

35. Ii. X.: Alhborn. H. Space, B.: Moore. P. B.: Zhou, Y.: Constantine. S.: Ziegler. L. D. J. Chem. Phys. 2000. 112, 4186.

36. Ji. X: Alhborn. H.: Space. B.: Moore. P. B. J. Chem. Phys. 2000. $113(19) .8693$.

37. Keyes. T. J. Chent. Phns. 1997. 106. 46.
38. Kim. J: Keyes. T. Phys. Rev: E 2002, 65. 061102.

39. Jansen, T. I. C.: Snijders. J. G.: Duppen. K. J. Chem. Phys. 2000. $113(1) .307$

40. Jansen. T. I. C.: Snijders. J. G.: Dupper1. K. . Chem. Phus. 2001. $11+.10910$.

41. Jansen, T. 1. C.: Duppen, K.: Snijders, J. G. Phns Ren: B 2012, 67. 134206.

42. Geiger. L. C.: Ladanyi, B. M. J. Chem. Phys. 1987,87, 191

43. Theory of Electric Polarization. Böttcher. C. J. F.: Bordewijk. P.: Elsevier: Amsterdam. 1978.

44. Solid State Phnsics: Asheroft. N. W.: Mermin. N. D.: Saunders College: Philadelphia. 1987.

45. Thole. B. T. Chem. Phys. 1981. 59. 341.

46. van Duijnen, P. Thi; Swart. M. J. Phys. Chem. A 1998. 102, 2399.

47. Jansen1. T. 1. C.: Swart. M.: Tensen. L: van Duijnen. P. Th.: Stiijders. T. G.: Duppen. K. J. Chem. Plhs. 2002. 116.3277.

48. Boeijenga. N. H.: Pugzlys. A.: Jansen. T. 1. C.: Stiijders. .T G.: Duppen. K. J. Chent Phys 2002. 117. 1181.

49. Muliamel, S.; Khidekel, V: Chernyak, V. Phss Ren: E 1996, 53 , R1.

50. Kiyohara. K.: Kamada. K.: Ohta. K. J. Chem. Phys. 2000. 112(14). 6338.

51. Keyes. T: Fourkas. T. T. J. Chem. Plas 2000. 112. 287.

52. Stone. A. J. The Theory of Internotecular Forces Voltme 32 of Intentational Series of Monographs on Chemisty, Oxford Unversity Press: Oxford. 1996.

53. Tildesley. D. J.: Madden. P. A. Mol. Phus. 1981. 42. 1137.

54. Berendsen. H. J. C.: Postma. J. P. M.: van Gunsteren. W. F.: DiNola. A.: Haak. J. R. J. Chent Phys. 1984. 81.3684.

55. Kubarych, K. J Milne, C. J Lin, S.; Miller, R. J. D. In $13 h$ International Conference on Cltrafast Phenomena, volume "XII" of Ultrafast Phenomena: Springer Verlag: Vancouver. 2002.

56. Kubarych. K.: Milne. C. J.: Lin1. S.: Miller. R. T. D. Appl. Phys. $B$ 74. Sion.

57. Saito. S. Private communication. 20002 